\title{
Characteristics of Patient with Macular Hole After Pars Plana Vitrectomy (PPV) and Internal Limiting Membran (ILM) Peeling in Cicendo Eye Hospital National Eye Center, Bandung
}

Rani Pitta, Rova Virgana, Iwan Sovani, Arief Kartasasmita, Erwin Iskandar, Grimaldi Ihsan, Made Indra Departement of Opthamology, Faculty of Medicine Universitas Padjadjaran Cicendo Eye Hospital National Eye Center

\section{ABSTRACT}

Introduction : Macular hole $(\mathrm{MH})$ can cause severe visual disturbance, but remarkable progress has been achieved in surgical treatment for eyes with this condition. Vitrectomy with internal limiting membrane (ILM) peeling allows a very high success rate for $\mathrm{MH}$ closure (approaching 90\%). To compare characteristics patients with closed and unclosed macular hole after pars plana vitrectomy (PPV) surgery and internal limiting membran (ILM) peeling.

Method : This was retrospective study which data was obtain from patient's medical records who underwent PPV and ILM peeling since July 1st to December 31th 2018.

Results : 27 eyes from 25 patients had $\mathrm{MH}$ surgery. 20 eyes (74\%) had closed $\mathrm{MH}$ and 7 (26\%) eyes unclosed $\mathrm{MH}$ after surgery. HFF value before surgery was $1,34 \pm 0,90$ and $\mathrm{MHI}$ was $1,09 \pm 0,81$ in closed $\mathrm{MH}$. Meanwhile in unclosed $\mathrm{MH}$, HFF value before surgery $0,53 \pm 0,12$ and $\mathrm{MHI}$ was $0,75 \pm 0,10$. $\mathrm{MHI} \geq 0,5$ and HFF value $\geq 0,9$ had a good prognostic factor.

Conclusion : Despite of good prognostic factor from OCT measurement and achieve anatomical success, foveal microstructure also important in visual recovery after $\mathrm{MH}$ surgery.

\footnotetext{
Keywords: Macular Hole, ILM Peeling, OCT macula.

Cite This Article: OMAS, Rani Pitta. The Characteristics of Patient with Macular Hole After Pars Plana Vitrectomy (PPV) and Internal Limiting Membran (ILM) Peeling in Cicendo Eye Hospital National Eye Center, Bandung. International Journal of Retina, [S.I.], v. 2, n. 1, feb. 2019. ISSN 2614-8536. Available at: <https://www.ijretina.com/index.php/ijretina/article/view/61>.
}

\section{${ }^{*}$ Correspondence to:}

Rani Pitta,

Department of Ophthalmology, Universitas Padjadjaran, rani_pitta@yahoo.co.id

\section{INTRODUCTION}

A macular hole was first described by Knapp in 1869. A macular hole (MH) is a full-thickness or partial-thickness defect in the macular region, and its pathogenesis can be idiopathic or result from myopia, trauma, or other causes. In most cases it is idiopathic, due to abnormal vitreofoveal traction. Idiopathic macular holes occur at a rate of approximately 8 per 100,000 persons per year and have a female-to-male ratio of 2 to 1 . The hallmark complaint of idiopathic macular hole formation is acute or subacute painless development of central visual distortion or blurred vision. ${ }^{1-3}$

The Gass classification system explains the clinically observed appearance of macular holes in four stages and their precursor lesions. Kelly and Wende were the first to report that vitreous surgery can improve the visual acuity in some eyes with acute, idiopathic macular holes. Since then, vitrectomy for idiopathic macular holes rapidly has become a widely performed procedure throughout the world. Recent studies confirm that high rates of anatomic success (between 85\% and 100\%) are achievable in large series of macular holes of all stages treated with vitrectomy, with approximately two-thirds of eyes achieving 20/50 or better vision. 1,3-4

Surgery for $\mathrm{MH}$ has undergone great developments since Kelly and Wendel first applied vitrectomy to treat $\mathrm{MH}$. Both closure rate and visual recovery have improved dramatically; internal limiting membrane (ILM) peeling in particular has significantly improved the closure rate. 
The use of dyes and the development of microincision surgery have reduced both the duration of surgery and the risk of damage from surgery. 1,3,5

Before the introduction of vitrectomy to treat $\mathrm{MH}$, the spontaneous closure rate for Gass stage 3 and $4 \mathrm{MHs}$ was merely $4 \%$, while that for stage $2 \mathrm{MHs}$ was $11.4 \%$ Following the introduction of vitrectomy by Kelly and Wendel, the closure rate increased to $58 \%$. As surgical techniques and instrumentation have improved, the closure rate has increased to as high as $90 \%$. The purpose of this study was compared characteristics patients with closed and unclosed macular hole after pars plana vitrectomy (PPV) surgery and internal limiting membran (ILM) peeling $2,3,5$

\section{METHOD}

This was a retrospective observational study involving patients who visit and underwent $\mathrm{MH}$ surgery from Vitreoretinal Unit Cicendo Eye Hospital, Bandung, Indonesia, between July $1^{\text {st }}$ to December $31^{\text {st }}, 2018$. Inclusion criteria were patients with macular hole after pars plana vitrectomy and ILM peeling. Macular hole was graded according of Gass criteria stage 3-4 and etiology $\mathrm{MH}$ was idiopathic. Exclusion criteria was macular hole with combination retinal detachment (MHRD), previous retina surgery and uncomplete data OCT.

In a retrospective chart research, we obtained preoperative data on onset, history of duration of symptom and visual acuity examination was done using snellen's Chart that converted to LogMar, lens status, indirect opthalmoscope with $+20 \mathrm{D}$ lens was used to evaluated the detailed fundus to examine extent and macular status. OCT macula was used to evaluated. Intraoperative data is the type of tamponade (silicone oil or gas) and postoperative data was visual acuity and OCT macula. We evaluate macular condition after surgery.

The pars plana vitrectomy with 23 gauge trocars using noncontact wideangle viewing system. Trocars were placed $3-4 \mathrm{~mm}$ from limbus that allows peripheral vitrectomy to be performed without touching the lens, and also switching between the 3 entry sites. A core vitrectomy was performed and the internal limiting membrane was removed with staining. The posterior hyaloid was elevated and trimmed in all patients. A fluidgas exchange was carried out, and the vitreous was filled with an inert air, gas (SF6 or C3F8) or fluid.

All patient were operated by five experienced posterior segment surgeons and postoperative follow-ups were made at the Vitreoretinal Unit Cicendo Eye Hospital National Eye Center, Bandung, Indonesia. All of this patient medical records that used for this study has already approved by Cicendo Hospital Medical Comittee. Data in this study was analyzed using Microsoft Excel 2016.

\section{RESULT}

The total of 27 eyes from 25 patients had MH surgery (pars plana vitrectomy and ILM peeling). Baseline characteristics in this study was described in Table 1.

The mean age of this study was $62 \pm 6,92$ years old with sex occur distributin of this study female $76 \%$ and male $24 \%$.

Indications for surgery includes stages 3 and 4 regarding to Gass classification. We examined stage of macular hole. There are 6 eyes (22\%) with stage III and 21 eyes $(78 \%)$ with stage 4 . There were 7 eyes (33\%) of stage IV didn't closed after surgery.

Table 1. Baseline Characteristic

\begin{tabular}{|c|c|c|c|}
\hline Characteristic & Mean \pm SD & $n(\%)$ & Range \\
\hline \multicolumn{4}{|l|}{ Sex } \\
\hline Male & & $6(24 \%)$ & \\
\hline Female & & 19 (76\%) & \\
\hline Age & $62 \pm 6,92$ & & $46-76$ y.o \\
\hline $40-49$ y.o & & $1(4 \%)$ & \\
\hline $50-59$ y.o & & $8(32 \%)$ & \\
\hline $60-69$ y.o & & $14(56 \%)$ & \\
\hline$>70$ y.o & & $2(8 \%)$ & \\
\hline \multicolumn{4}{|l|}{ Lens Status } \\
\hline Phakic & & $21(78 \%)$ & \\
\hline Pseudophakic & & $6(22 \%)$ & \\
\hline \multicolumn{4}{|c|}{ Stage Macular Hole } \\
\hline III & & $6(22 \%)$ & \\
\hline IV & & $21(78 \%)$ & \\
\hline
\end{tabular}


We examined visual acuity in patients after macular surgery (PPV + ILM peeling). There were 20 eyes (74\%) had closed $\mathrm{MH}$ and 7 eyes (26\%) unclosed $\mathrm{MH}$ after the surgery. For closed $\mathrm{MH}$, mean uncorrected VA (visual acuity) before surgery was $0.73 \pm 0.24$ Log and after surgery $0,65 \pm 0,25$ Log. For unclosed $\mathrm{MH}_{\text {, mean }}$ uncorrected VA before surgery was 1,30 $\pm 0,31 \mathrm{Log}$ and after surgery $1,165 \pm 0,165 \quad$ Log.

There were 27 eyes underwent pars plana vitrectomy and ILM peeling. There were 19 eyes used SF6 as a tamponade and 1 eye with air in closed $\mathrm{MH}$. There were 6 eyes used SF6 as a tamponade in unclosed $\mathrm{MH}$.

We also examined MHI (macular hole index) and HFF (hole form factor) from OCT macula patients as a prognostic factor. If $\mathrm{MHI}$ value $\geq 0.5$ and $\mathrm{HFF} \geq 0,9$, the patient has a good prognosis. We found mean HFF value before surgery was $1,34 \pm 0,90$ and $\mathrm{MHI}$ was $1,09 \pm 0,81$ in closed $\mathrm{MH}$. Meanwhile in unclosed $\mathrm{MH}, \mathrm{HFF}$ value before surgery $0,53 \pm 0,12$ and $\mathrm{MHI}$ was $0,75 \pm 0,10$.

\section{DISCUSSION}

A macular hole is an anatomic discontinuity of the neurosensory retina that develops in the center of the macula or fovea. Typically, the patient will experience metamorphopsia and decreased visual acuity. Most investigators believe that macular holes are caused by pathologic vitreoretinal traction at the fovea. ${ }^{1-4}$

Tabel 2. Characteristics After Macular Surgery

\begin{tabular}{llclc}
\hline Characteristics & Mean \pm SD & n (eyes) & Mean \pm SD & n (eyes) \\
\hline & Closed MH & & Unclosed MH & \\
Total & & 20 & & 7 \\
Duration of symptoms & $8,34 \pm 7,43$ & & $11,42 \pm 5,42$ & \\
Uncorrected VA before surgery & $0,73 \pm 0,24$ & & $1,30 \pm 0,31$ & \\
Uncorrected VA after surgery & $0,65 \pm 0,25$ & & $1,165 \pm 0,165$ & \\
Follow up (weeks) & $9,65 \pm 7,76$ & & $4 \pm 0,00$ & \\
OCT Macula & & & & \\
Stage MH Pre-op & & 6 & & \\
III & & 14 & \\
IV & & & $0,53 \pm 0,12$ & \\
HFF (Hole form factor) & $1,34 \pm 0,90$ & & $0,75 \pm 0,10$ & \\
MHI (Macular Hole Index) & $1,09 \pm 0,81$ & & & 7 \\
Tamponade & & 19 & \\
SF6 & & 1 & \\
air & & & \\
\hline
\end{tabular}

The Beijing Eye Study is a population-based crosssectional study of 4346 subjects that found a prevalence of macular holes of $0.09 \pm 3.04 \%$. Eye Disease CaseControl Study Group wrote the majority (72\%) of idiopathic macular holes occurred in women; more than $50 \%$ of holes were found in individuals 65 to 74 years of age and only $3 \%$ in those under the age of 55 . In this study, mean age of was $62 \pm 6,92$ years old with sex distributin of this study 6 male (24\%) and 19 female $(76 \%)^{1,-4}$

The duration of a patient's symptoms is an important predictor of anatomic macular hole closure and visual improvement. Kelly and Wendel reported that visual outcomes were best for those with symptoms existing for less than 6 months. In this study, duration of symptoms mean 8,34 \pm 7,43 weeks for closed $\mathrm{MH}$ and 11,42 $\pm 5,42$ weeks for unclosed $\mathrm{MH}^{2-4}$
For decades, macular holes (MH) have been classified in four stages, as first described by Donald Gass in 1988. Williamson et al reported that among 351 cases, the stage 2,3 , and 4 closure rates were $95.8 \%, 73.0 \%$, and $56.3 \%$, respectively, and this difference was significant. In this study, 7 eyes with unclosed $\mathrm{MH}$ were stage IV MH.,3-6

Optical coherence tomography (OCT) has enhanced our understanding of $\mathrm{MH}$ by providing an objective and reproducible way of visualizing the macula. OCT has revolutionized macular imaging and also allows quantitative measurements of many anatomic parameters. The anatomic parameter could be used in prognostic factor in macular hole surgery. The anatomic parameter can help predicted postoperative anatomic and visual outcome. The diameter of the macular hole measured by OCT at the level of the retinal pigment epithelium and the minimum diameter seem to provide a 
prognostic factor for postoperative visual outcome and anatomical success of macular hole surgery. ${ }^{6-8}$

The hole form factor (HFF) is the first calculated OCT index used as a prognostic factor. The HFF is the quotient of the summation of the left and right arm lengths divided by the basal hole diameter. Puliafito et al found an $80 \%$ anatomical success rate in patients with HFF greater than 0.9 . HFF value $\geq 0.9$ had better prognosis. In this study mean HFF value in closed $\mathrm{MH}$ was 1,34 $\pm 0,90$ before surgery $(n=20)$ and in unclosed MH HFF value was $0,53 \pm$ $0,12(n=7) .^{6-8}$

$\mathrm{MH}$ index $(\mathrm{MHI})$ is an intuitive predictor for visual outcome following $\mathrm{MH}$ surgery. The $\mathrm{MHI}$ is defined as the ratio of the hole height to the basal hole diameter and is reported to be positively correlated to the postoperative visual acuity in several studies. MHI value $\geq 0.5$ had better visual acuity than those with an $\mathrm{MHI}$ value $<0.5$. In this study, we found that $\mathrm{MHI}$ value in closed $\mathrm{MH}$ before surgery was $1,09 \pm 0,81 \mu \mathrm{m}(\mathrm{n}=20)$ and compare to unclosed MHI 0,53 $\pm 0,15 \mu \mathrm{m}(\mathrm{n}=7)$ after surgery. ${ }^{7-9,10}$

The ILM is the basal lamina of the inner retina and is thought to be a scaffold for proliferation of fibrocytes, myofibroblasts, and retinal pigment epithelial cells. ILM peeling increases the likelihood of successful macular hole closure, but, because the ILM also plays a role in the structural integrity of the retina, it has been postulated that removal of this membrane could be functionally damaging to the retina. $3,7,10$

Cochrane review found that ILM peeling achieves higher anatomical success with a reduced need for additional surgical interventions when compared to nonpeeling in treating patients at stages 3 , and 4 . In this study, there were 27 eyes underwent vitrectomy and ILM peeling and only 1 eye need additional surgical intervention because of re-open. We found in OCT MHI value : 0,23 and HFF 0,426 from this patient (reopen). ${ }^{3.6,10}$

Kelly and Wendel reported that $73 \%$ patients who underwent vitrectomy resulting in successful macular reattachment experienced an improvement in visual acuity of two lines or better. In this study, VA not improve enough. We analyze because patients didn't get corrected VA, some patients had cataract, unclosed $\mathrm{MH}$, or in closed $\mathrm{MH}$ still had irregular closure so it can limited visual recovery. ${ }^{6,8,10}$

Photoreceptor defects were correlated with postoperative BCVA. There are four distinct hyperreflective lines that can be viewed by SD-OCT: the photoreceptor inner segment/outer segment (IS/OS) junction, the external limiting membrane (ELM), the cone outer segment tips (COST), and the retinal pigment epithelium (RPE). The International Nomenclature OCT Consensus refers to the IS/OS junction as the ellipsoid zone. It has also been demonstrated that the integrity of the ELM and ellipsoid zone is the most important factor related to postoperative visual acuity. $6,8,10$
Retinal tamponade may be created using different agents at the conclusion of macular hole surgery to achieve anatomic closure of the macular hole. Tamponade options include the use of air (days), SF6 (2 to 4 weeks), C3F8 (1 to 3 months), or silicone oil (long term). Commonly used long-lasting gases include sulfur hexafluoride (SF6) and perfluoropropane (C3F8). No significant differences in anatomic success or visual outcomes have been reported between these. In this study we found that 19 eyes used SF6 and 1 eye using air in closed $\mathrm{MH}$. In unclosed $\mathrm{MH}$, there were 7 eyes used SF6..$^{3,10-11}$

\section{CONCLUSION}

Macular hole (MH) is a round full-thickness opening in the foveal center. Macular hole surgery consists of vitrectomy with Internal Limiting Membrane (ILM) Peeling. ILM peeling for macular holes rapidly has high rates of anatomic success are achievable. Despite of good prognostic factor from OCT measurement and achieve anatomical success, foveal microstructure also important in visual recovery after $\mathrm{MH}$ surgery.

\section{REFERENCE}

1. Ryan SJ. Retina. Sixth Ed. China: Elsevier; 2018. 557-68

2. Cantor.B. American academy of opthalmology. basic and clinical science course. Retina and vitreous. 2016-2017

3. Olsen T.W. et al. Idiopathic macular hole : retina/vitreous preferred practice pattern development process and participants. 2014. 1-33

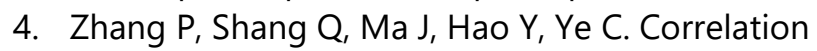
between postoperative area of high autofluorescence in macula and visual acuity after macular hole closure. European Journal of Ophthalmology. 2017. 781-5

5. Kang SW. Types of macular hole closure and their clinical implications. British Journal of Ophthalmology. 2013. 1015-9.

6. Zhao P, Wang S, Liu N, Shu Z, Zhao J. A review of surgical outcomes and advances for macular holes. 2018. Hal 1-10

7. Goldberg RA, Waheed NK, Duker JS. Optical coherence tomography in the preoperative and postoperative management of macular hole and epiretinal membrane. British Journal of Ophthalmology. 2014. 20-3

8. Tao LW, Wu Z, Guymer RH, Luu CD. Ellipsoid zone on optical coherence tomography: a review: Ellipsoid zone on optical coherence tomography. Clinical \& Experimental Ophthalmology. 2016. 422-30 
9. J. R, C. R. Application of optical coherence tomography and macular holes in ophthalmology. Optical coherence tomography. Intech. 2013

10. Rizzo s, et al. Internal limiting membrane peeling versus inverted flap technique for treatment of full-thickness macular holes: a comparative study in a large series of patients. Retina. 2018. 73-8.
11. Casini G, Loiudice P, De Cillà S, Radice P, Nardi M. Sulfur hexafluoride (SF6) versus perfluoropropane (C3F8) tamponade and short term face-down position for macular hole repair: a randomized prospective study. International Journal of Retina and Vitreous. 2016. 1-6

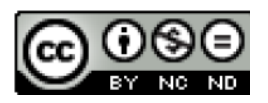

This work licensed under Creative Commons Attribution 